\title{
Tecnologías de aprovechamiento energético de biomasa para la electrificación rural en cundinamarca
}

Juan Sebastián Sánchez-Gómez ${ }^{1}$

Universidad de los Andes, Colombia

js.sanchez14@uniandes.edu.co

Juan Manuel Fajardo-Pinilla²

Universidad Minuto de Dios

jfajar25@gmail.com

DOI: https://doi.org/10.21158/23823399.v7.n0.2019.2643

Fecha de recepción: 12 de marzo de 2020

Fecha de aprobación: 14 de julio de 2020

Cómo citar este artículo: Sánchez-Gómez, J. S.; Fajardo-Pinilla, J. M. (2019). Tecnologías de aprovechamiento energético de biomasa para la electrificación rural en Cundinamarca. Revista Ontare, 7, 117-132. DOI: https://doi.org/10.21158/23823399.v7.n0.2019.2643

${ }^{1}$ Pedagogo del Centro de Innovación en Tecnología y Educación de la Universidad de los Andes. Ingeniero Biomédico, Magíster en Educación y Magíster en Políticas Públicas de la Universidad de los Andes. ORCID: https://orcid.org/0000-0001-5199-7486

${ }^{2}$ Químico de la Universidad Nacional y Especialista de Diseño de Ambientes de Aprendizaje de la Universidad Minuto de Dios. ORCID: https://orcid.org/0000-0002-4109-0693 


\section{RESUMEN}

En el 2019, el Instituto de Planificación y Promoción de Soluciones Energéticas para las Zonas no Interconectadas (IPSE) reportó 1710 zonas rurales con 128 587 personas que acceden a energía eléctrica entre cuatro y doce horas por día. Este acceso limitado a electricidad genera inseguridad y altos costos en mantener en funcionamiento las plantas eléctricas, con inversiones que superan los cien dólares diarios para generar energía con diésel en municipios con ingresos bajos. Este déficit energético en Colombia es una oportunidad para la generación de electricidad a partir de energías renovables como, por ejemplo, la biomasa, las cuales podrían superar las condiciones geográficas que dificultan la infraestructura eléctrica convencional en las zonas rurales colombianas. Este artículo de revisión presenta los avances y las tendencias sobre las tecnologías de aprovechamiento energético de biomasa en las zonas rurales de Cundinamarca. El objetivo de esta revisión es presentar las principales tecnologías implementadas en Cundinamarca sobre el aprovechamiento energético de la biomasa a partir de biodigestión anaerobia. La revisión incluye proyectos productivos que se han desarrollado mediante el diseño y la implementación de biodigestores de bajo costo. Estos proyectos de ingeniería sostenible, desde el año 2000, han permitido empoderar a las comunidades rurales mediante la transferencia de tecnología y la promoción de economías circulares basadas en el emprendimiento y la mejora de la competitividad regional; por eso el texto plantea la necesidad de un futuro estudio ambiental que determine el impacto de estos proyectos productivos sobre los ecosistemas que predominan en estos ambientes rurales.

Palabras clave: energía rural renovable; generación de electricidad; acceso energético; electrificación rural; biodigestor; biomasa; transferencia de tecnología; economías circulares. 


\section{Biomass energy technologies for rural electrification in Cundinamarca}

\section{ABSTRACT}

In 2019, the Institute for the Planning and Promotion of Energy Solutions for Non-Interconnected Areas (IPSE by its abbreviation in Spanish) reported 1710 rural areas with 128,587 people accessing electricity between four and twelve hours per day. This limited access to electricity generates insecurity and high costs for maintaining the power plants in operation, with investments exceeding one hundred dollars a day for generating energy using diesel in low-income municipalities. This energy deficit in Colombia is an opportunity for generating electricity from renewable energies such as biomass, which could overcome the geographical conditions that hinder conventional electrical infrastructure in the rural areas in Colombia. This review article presents the advances and trends in biomass energy technologies in the rural areas of Cundinamarca. The aim of this review is to present the main technologies that have been implemented in Cundinamarca on the energetic use of biomass from anaerobic biodigestion. The review includes productive projects that have been developed through the design and implementation of low-cost biodigesters. Since 2000 , these sustainable engineering projects have empowered rural communities via the transfer of technology and the promotion of circular economies based on entrepreneurship and the improvement of regional competitiveness. For this reason, the text poses the need for a future environmental study to determine the impact of these productive projects on the ecosystems that predominate in these rural environments.

Keywords: renewable rural energy; generation of electricity; energy access; rural electrification; biodigester; biomass; technology transfer; circular economies. 


\section{Tecnologias de aproveitamento energético de biomassa para a eletrificação rural em Cundinamarca}

\section{RESUMO}

Em 2019, o Instituto de Planejamento e Promoção de Soluções Energéticas para as Zonas não Interconectadas (IPSE) reportou 1710 zonas rurais com 128.587 pessoas que acedem a energia elétrica entre quatro e doze horas por dia. Este acesso limitado a eletricidade gera insegurança e altos custos em manter em funcionamento os geradores elétricos, com investimentos que superam os cem dólares diários na produção de energia com diesel em municípios com baixas receitas. Este déficit energético na Colômbia é uma oportunidade para a geração de eletricidade a partir de energias renováveis como, por exemplo, a biomassa, as quais poderiam superar as condições geográficas que dificultam a infraestrutura elétrica convencional nas zonas rurais colombianas. Este artigo de revisão apresenta os avanços e as tendências sobre as tecnologias de aproveitamento energético de biomassa nas zonas rurais de Cundinamarca. 0 objetivo desta revisão é apresentar as principais tecnologias implementadas em Cundinamarca sobre o aproveitamento energético da biomassa a partir de biodigestão anaeróbia. A revisão inclui projetos produtivos que se desenvolveram mediante o desenho e a implementação de biodigestores de baixo custo. Estes projetos de engenharia sustentável, desde o ano 2000, têm permitido o empoderar às comunidades rurais mediante a transferência de tecnologia e a promoção de economias circular baseadas no empreendimento e na melhoria da competitividade regional. Por isso o texto propõe a necessidade de um futuro estudo ambiental que determine o impacto destes projetos produtivos sobre os ecossistemas que predominam nestes ambientes rurais.

Palavras-chave: energia rural renovável; geração de eletricidade; acesso energético; eletrificação rural; biodigestor; biomassa; transferência de tecnologia; economias circular. 


\section{Technologies utilisant la biomasse pour la production d'énergie électrique en milieu rural dans département de Cundinamarca}

\section{RÉSUMÉ}

En 2019, l'Institut pour la planification et la promotion des solutions énergétiques pour les zones non interconnectées (IPSE) a signalé 1710 zones rurales comptant plus de 128.000 personnes n'ayant qu'un accès restreint à l'énergie électrique - entre quatre et douze heures par jour. Cet accès limité à l'énergie génère insécurité et coûts élevés de manutention des centrales en fonctionnement, occasionnant des dépenses supérieures à cent dollars par jour pour la production d'électricité au diesel dans les municipalités à faible revenu. Ce déficit énergétique est une opportunité pour la production d'électricité à partir d'énergies renouvelables comme, par exemple, la biomasse, qui permettrait de surmonter les difficultés géographiques limitant la construction d'infrastructures électriques conventionnelles dans ces zones rurales. Cet article de synthèse présente les progrès et tendances technologiques utilisant l'énergie de la biomasse dans les zones rurales du département de Cundinamarca. Cette étude présentera également les principales technologies mises en œuvre en Cundinamarca pour l'utilisation énergétique de la biomasse issue de la biodigestion anaérobie et analysera des projets productifs développés grâce à la conception et mise en place de biodigesteurs à faible coût. Ces projets d'ingénierie durable, ont permis, depuis les années 2000 , de responsabiliser les communautés rurales par le transfert de technologie et la promotion d'économies circulaires fondées sur l'entrepreneuriat et l'amélioration de la compétitivité régionale. Enfin, ce texte soulève l'importance et la nécessité d'une étude environnementale future pour déterminer l'impact de ces projets productifs sur les écosystèmes de ces milieux ruraux.

Mots clés: énergie renouvelable, ruralité; production d'électricité; accès à l'énergie; électrification rurale; biodigesteur; biomasse; transfert de technologie; économies circulaires. 


\section{Introducción}

El acceso a la energía eléctrica es necesario para el desarrollo económico y el bienestar de los seres humanos. Sin embargo, el constante incremento del consumo energético a nivel mundial en los últimos años, sumado al crecimiento poblacional, deja en evidencia la necesidad de reducir la dependencia a los combustibles fósiles, así como de promover el estudio y el uso potencial de la biomasa como fuente de energía renovable. En este sentido, el objetivo del presente artículo de revisión es presentar las principales tecnologías implementadas en Cundinamarca sobre el aprovechamiento energético de la biomasa a partir de biodigestión anaerobia. Para comenzar, se introducen las energías renovables mediante estadísticas mundiales y nacionales sobre su uso como alternativa a las fuentes de energía tradicionales. A continuación, se presentan cifras de electrificación rural y el Atlas del potencial energético de la biomasa residual en Colombia. Posteriormente, se presentan estudios relevantes sobre la producción de biogás mediante biodigestión anaerobia en Colombia y proyectos rurales para la generación de biogás implementados en Cundinamarca.

\subsection{Energías renovables en el mundo}

Según la International Energy Agency (IEA), desde 1990 las fuentes de energía renovable han tenido un crecimiento del $2 \%$, lo cual resulta mayor a la tasa de crecimiento mundial que alcanzó el 1,7 \% (véase la Figura 1). La tercera tasa de crecimiento correspondió al biogás con $11,9 \%$, y los biocombustibles líquidos con el 9,7\%. En el 2017 las energías renovables fueron el segundo mayor contribuyente a la producción mundial de electricidad con un $24,5 \%$, después del carbón. En este mismo año, 1200 millones de personas no accedieron a energía eléctrica y 2700 millones usan biomasa para cocinar (IEA, 2017). 
Figura 1. Crecimiento anual mundial de energías renovables de 1990-2017

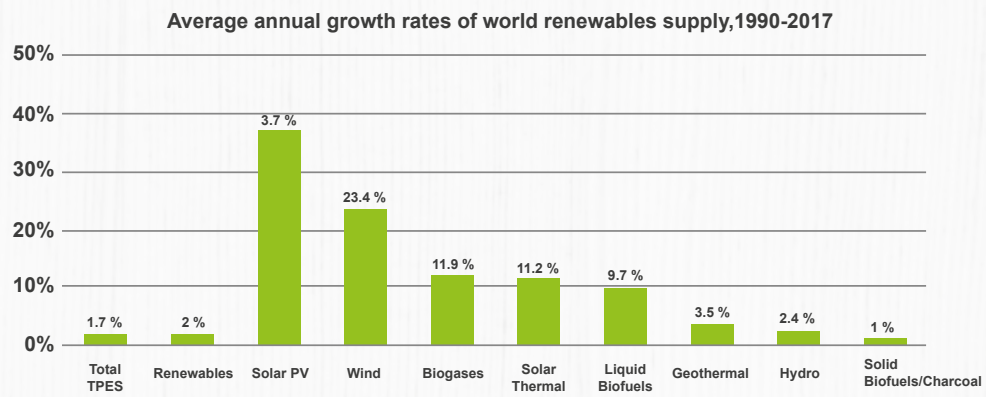

Fuente. IEA, 2017.

\subsection{Electrificación rural en Colombia}

Según el artículo 1 de la Ley 855 de 2003, las zonas no interconectadas (ZNI) son aquellas zonas que no están conectadas al Sistema Interconectado Nacional, reportadas por el Instituto de Planificación y Promoción de Soluciones Energéticas para las Zonas No Interconectadas (IPSE). El IPSE tiene como misión identificar, estructurar, implementar y monitorear las soluciones energéticas sostenibles, con el fin de mejorar la calidad de vida de los habitantes de las ZNI (IPSE, 2018).

Las ZNI representan el $51 \%$ del territorio colombiano (véase la Figura 1), lo cual incluye 18 departamentos, cinco capitales departamentales, 27 cabeceras municipales, 95 municipios y 1789 localidades. En estas zonas ZNI se atienden 194712 usuarios mediante 91 entes prestadores de servicios energéticos, con una capacidad operativa de $241634 \mathrm{~kW}$, de la cual el 96,6\% son fuentes de energía no renovable y el 3,4 \% de energías renovables (IPSE, 2018).

En estas zonas ZNI, una gran proporción de la población usa gas propano como combustión para cocinar. Sin embargo, el gas propano tiene un alto costo mensual de compra -USD 50-y transporte -USD 25-, así como existe riesgo en su gestión y almacenamiento. Por tal motivo, la digestión anaerobia surge como una alternativa de energía renovable para las zonas ZNI (CastroMolano et al., 2017), tal como se puede evidenciar en el Atlas del potencial energético de la biomasa residual en Colombia (SI3EA, 2010). 


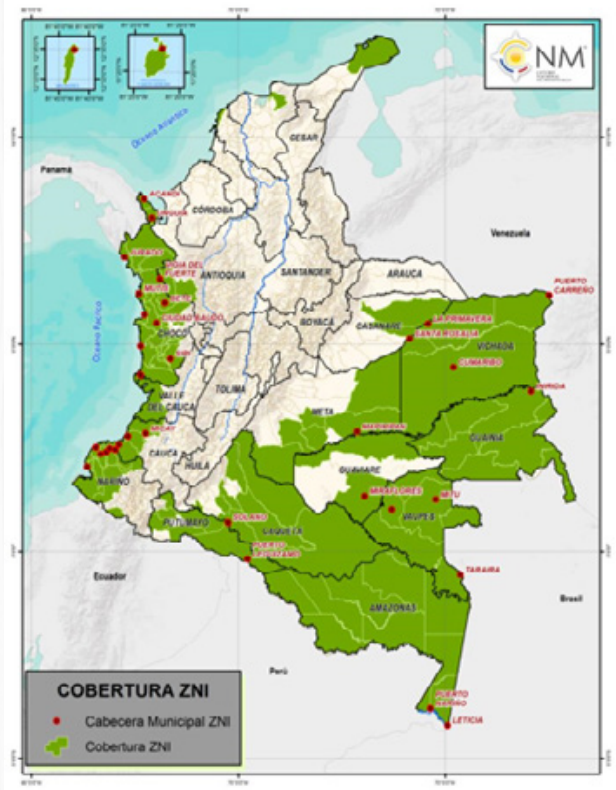

Fuente. IPSE, 2018.

\subsection{Atlas del potencial energético de la biomasa residual en Colombia}

Este atlas analiza la oferta energética de la biomasa que está contenida en las especies más representativas de los sectores agrícola, pecuario y de los residuos orgánicos urbanos. Las fuentes de biomasa residual, en el sector agrícola, son los residuos agrícolas de cosecha y los residuos agroindustriales; en el sector pecuario son el estiércol bovino, porcino y avícola; y en el sector de los residuos sólidos orgánicos urbanos son los residuos de las plazas de mercado, de los centros de abastos y de las podas. Así mismo, el atlas reporta el potencial energético de la biomasa residual en los sectores estudiados mediante modelos matemáticos que desarrollan una función de la masa del residuo y de su contenido energético - calculado a través del poder calórico inferior-. Con todo lo anterior, se obtienen mapas que representan gráficamente las variables cantidad de área cultivada y población, potencial energético y rendimiento neto de energía de la biomasa residual (SI3EA, 2010). 


\section{Producción de biogás a partir de biodigestión anaerobia}

Cabe resaltar que una gran proporción de los estudios reportados de generación de biogás en Colombia hacen referencia al empleo de biomasas derivado de actividades agropecuarias mediante técnica de conversión biológica -biodigestión anaeróbica-. Parra, Botero y Botero (2019) afirman que el biogás producido por la digestión anaerobia de residuos pecuarios es una solución para la sustitución de la biomasa tradicional usada en iluminación y calor. Su uso presenta ventajas tales como la mejora en la productividad de las fincas, la reducción del impacto ambiental y el rompimiento de la dependencia a los combustibles fósiles y agroquímicos. Marín, Salazar, Castro y Escalante (2016), por su parte, trabajan un proceso de codigestión de vinaza y gallinaza de jaula como una alternativa de manejo y estabilización de residuos generados por la agroindustria colombiana. Mientras que Fernández, Martínez, Morán y Gómez (2016) se fijan en el lactosuero como subproducto del proceso de elaboración del queso en la producción de biocombustibles.

Entre las alternativas disponibles de aprovechamiento energético se encuentra la digestión anaerobia, la cual es una de las tecnologías más empleadas en el tratamiento de diversos tipos de residuos. Castro, Escalante, Gómez y Jiménez (2016) registran el potencial de biometanización para las aguas residuales de una planta de sacrificio bovino que se determinó para las temperaturas de operación de $37^{\circ} \mathrm{C}$ y $30^{\circ} \mathrm{C}$ con estiércol bovino preincubado como fuente de inóculo. Así mismo, Cendales y Jiménez (2014) llevan a cabo la simulación computacional del proceso de digestión anaeróbica de la mezcla homogeneizada de estiércol bovino y residuos cítricos con el fin de evaluar la viabilidad de la generación de energía renovable a partir de la codigestión de estos residuos.

Adicionalmente, Escalante, Guzmán y Castro (2014) reportan que el procesamiento agroindustrial de fique genera, aproximadamente, $20800 \mathrm{~kg}$ de residuos por hectárea sembrada en Colombia, lo que corresponde a jugo y bagazo que son descartados al ambiente, de modo que generan problemas 
de contaminación. En este trabajo se estudió la producción de biogás a partir del bagazo de fique empleando como inóculo una mezcla de líquido ruminal y estiércol de cerdo.

Nabarlatz, Arenas-Beltrán, Herrera-Soracá y Niño-Bonilla (2013) infieren que la materia prima más utilizada en Colombia para producir biodiesel es el aceite de palma, de manera que su producción ha aumentado drásticamente en los últimos años y genera efluentes con alta carga contaminante para el medio ambiente como consecuencia del proceso; dado que las características físico-químicas de estos efluentes son propicias para la producción de biogás mediante digestión anaerobia.

Finalmente, Núñez (2012) estudia la producción agropecuaria del Meta, considerando que la biomasa producida representa un problema desde el punto de vista ambiental debido a la falta de técnicas para su aprovechamiento y con una gran posibilidad de reusarse en la producción de energía para diferentes procesos, dentro de una producción agrícola, o a fin de generar energía para la agroindustria, supliendo el uso de energía proveniente del carbón, del diésel y de la gasolina, entre otros.

\section{Proyectos rurales de producción de biogás en Cundinamarca}

Por otra parte, un empleo interesante de los residuos agroindustriales y los residuos sólidos urbanos (RSU) en la región cundiboyacense, de acuerdo con los estudios reportados, es la generación de biogás para la producción de energía eléctrica, ya que reducen los efectos contaminantes y el impacto ambiental de estos. Tal como lo reportan Alzate, Restrepo y Jaramillo (2018), se estima el potencial de producción de energía eléctrica desde RSU con dos tecnologías de conversión: incineración -conversión térmica- y digestión anaerobia -conversión biológica-. 
Adicionalmente, Montenegro, Rojas, Cabeza y Hernández (2016) determinan el potencial de producción de biogás de los residuos agroindustriales en el departamento de Cundinamarca a través de digestión anaerobia, para los municipios de Fómeque, Guachetá y Anapoima. Rodríguez, Colmenares, Barragán y Mayorga (2017) se centran en el aprovechamiento energético de la biomasa generada por Eichhornia crassipes, una maleza acuática conocida popularmente con el nombre de buchón, lirio o violeta de agua, la cual se procesa con el propósito de obtener briquetas, bioetanol y biogás. Así mismo, describen los procesos para obtener estos derivados y realizan una comparación cualitativa entre ellos, por la cual presentan un panorama energético de la Eichhornia crassipes como fuente de energía alternativa y sostenible, situaciones que pueden ser similares para muchos municipios de la región dada su vocación agropecuaria y la deficiencia creciente en la disposición de dichos residuos.

Finalmente, según Acosta y Pasqualino (2014), en Colombia, en promedio, el $93 \%$ de los residuos sólidos se disponen en rellenos sanitarios adecuados, lo cual presenta un claro desaprovechamiento de materiales que son buena fuente de generación de energías alternativas renovables (EAR) y que, además, son negocios sustentables y de tecnología limpia.

\section{Biodigestión anaerobia a partir de residuos de alimentos preparados}

Por último, respecto a la implementación de biodigestores anaerobios alimentados con residuos de alimentos preparados, Solarte, Mariscal y Aristizabal (2017) evalúan la digestión y codigestión anaerobia de residuos de comida y de poda en biorreactores a escala de laboratorio, reportando que el deterioro ambiental provocado por el alto consumo de combustibles fósiles y la inadecuada disposición de residuos sólidos ha incentivado su conversión en productos energéticos de alto valor agregado a partir de diferentes procesos biotecnológicos con el fin de compensar parte de la demanda energética. Evalúan también la eficiencia, el rendimiento y la productividad del proceso de digestión y codigestión anaerobia de residuos de comida y poda a fin de valorar su viabilidad como sustratos en la generación de biogás. 
En este sentido, Rincón, Rincón, Mata y Chirinos (2014) estudian la biodegradabilidad de residuos de alimentos preparados bajo condiciones mesofílicas y termofílicas, utilizando un reactor anaeróbico de mezcla completa. Así mismo, realizan la digestión anaeróbica de residuos de alimentos preparados (RAP) en un reactor anaeróbico de mezcla completa (RAMC) de 3,5 L, con flujo discontinuo bajo condiciones mesofílicas (CM) a $37^{\circ} \mathrm{C}$, y termofílicas (CT) a 55 ${ }^{\circ} \mathrm{C}$. Su objetivo fue evaluar la biodegradabilidad de RAP con distintos lodos biológicos y tiempos de retención hidráulicos (TRH). Las mejores remociones y producción de biogás se generaron durante el TRH de 30 d en cm con el efluente de cachaza.

\section{Conclusiones}

Esta revisión permitió identificar las principales tendencias tecnológicas sobre el aprovechamiento energético de la biomasa en Colombia. Estas tendencias son un primer acercamiento a la producción de biogás en comunidades rurales, las cuales usan diseños empíricos para generar biodigestión anaerobia cuyos residuos en grandes cantidades son tóxicos para los humanos y contaminantes para el medio ambiente. Por tanto, se requiere como trabajo futuro un estudio ambiental que determine el impacto de estos proyectos productivos sobre los ecosistemas que predominan en estos ambientes rurales. Así mismo, es necesario darle a conocer a estas comunidades oportunidades de financiación, de transferencia tecnológica y de conocimiento con el fin de optimizar estos procesos de biodigestión, hacerlos más amigables con el medio ambiente y sostenibles a largo plazo. 


\section{Referencias}

Acosta, M.; Pasqualino, J. (2014). Potencial de uso de biogás en Colombia. Teknos Revista Científica, 14(2), 27-33. DOI: https://doi. org/10.25044/25392190.468

Alzate, S.; Restrepo, B.; Jaramillo, A. (2018). Electricity generation potential from solid waste in three Colombian municipalities. TecnoLógicas, 21(42), 111-128. DOI: https://doi.org/10.22430/22565337.782

Castro, L.; Escalante, H.; Gómez, O. J.; Jiménez, D. P. (2016). Análisis del potencial metanogénico y energético de las aguas residuales de una planta de sacrificio bovino mediante digestión anaeróbica. DYNA, 83(199), 41-43. DOI: https://doi.org/10.15446/dyna.v83n199.56796

Castro-Molano, L.; Escalante, H.; Jaimes-Estévez, J.; Díaz, L. J.; Vecino, K.; Rojas, G.; Mantilla, L. (2017). Low cost digester monitoring under realistic conditions: rural use of biogas and digestate quality. Bioresource Technology, 239, 311-317. DOI: https://doi.org/10.1016/j. biortech.2017.05.035

Cendales, E.; Jiménez, S. (2014). Modelamiento computacional de la producción de energía renovable a partir del biogás mediante la codigestión anaeróbica de la mezcla de residuos cítricos y estiércol bovino. Revista Escuela de Administración de Negocios, (77), 42-62. DOI: https://doi.org/10.21158/01208160.n77.2014.814

Escalante, H.; Guzmán, C.; Castro, L. (2014). Anaerobic digestion of fique bagasse: an energy alternative. DYNA, 81(183), 74-85. DOI: https://doi. org/10.15446/dyna.v81n183.34382

Fernández, C.; Martínez, E.; Morán, A.; Gómez, X. (2016). Procesos biológicos para el tratamiento de lactosuero con producción de biogás e hidrógeno. Revisión bibliográfica. Revista Investigación, Optimización y Nuevos Procesos En Ingeniería, 29(1), 47-62. DOI: https://doi.org/10.18273/revion. v29n1-2016004

IEA (International Energy Agency). (2017). Renewables statistics. Comprehensive historical review and current market trends in renewable energy. Recuperado de https://www.iea.org/statistics/renewables/ 
IPSE (Instituto de Planificación y Promoción de Soluciones Energéticas para las Zonas no Interconectadas). (2018). Informe de gestión 2017-2018. Recuperado de https://bit.ly/36dKlab

Ley 855 de 2003. (18 de diciembre de 2003). Por la cual se definen las Zonas no Interconectadas. Diario Oficial, núm. 45.405, Congreso de Colombia.

Marín, J. D.; Salazar, L.; Castro, L.; Escalante, H. (2016). Co-digestión anaerobia de vinaza y gallinaza de jaula: alternativa para el manejo de residuos agrícolas colombianos. Revista Colombiana de Biotecnología, 18(2), 6-12. DOI: $\underline{\text { https://doi.org/10.15446/rev.colomb.biote.v18n2.53853 }}$

Montenegro, K. T.; Rojas, A. S.; Cabeza, I.; Hernández, M. A. (2016). Potencial de biogás de los residuos agroindustriales generados en el departamento de Cundinamarca. Revista Ion, Investigación, Optimización y Nuevos Procesos en Ingeniería, 29(2), 23-37. DOI: https://doi.org/10.18273/revion.v29n2$\underline{2016002}$

Nabarlatz, D. A.; Arenas-Beltrán, L. P.; Herrera-Soracá, D. M.; Niño-Bonilla, D. A. (2013). Biogas production by anaerobic digestion of wastewater from palm oil mill industry. Ciencia, Tecnología y Futuro, 5(2), 73-84. DOI: https://doi.org/10.29047/01225383.58

Núñez, D. W. (2012). Uso de residuos agrícolas para la producción de biocombustibles en el departamento del Meta. Tecnura, 16(34), 142-156. DOI: https://doi.org/10.14483/udistrital.jour.tecnura.2012.4.a10

Parra, D.; Botero, M.; Botero, J. (2019). Biomasa residual pecuaria: revisión sobre la digestión anaerobia como método de producción de energía y otros subproductos. Revista UIS Ingenierías, 18(1), 149-160. DOI: https:// doi.org/10.18273/revuin.v18n1-2019013

Rincón, M.; Rincón, N.; Mata, J.; Chirinos, I. (2014). Biodegradabilidad de residuos de alimentos preparados bajo condiciones mesofílicas y termofílicas utilizando un reactor anaeróbico de mezcla completa. Ciencia e Ingeniería Neogranadina, 24(1), 29-48. DOI: https://doi.org/10.18359/ $\underline{\operatorname{rcin} .6}$

Rodríguez, A.; Colmenares, F.; Barragán, J.; Mayorga, M. (2017). Aprovechamiento energético integral de la Eichhornia Crassipes (buchón de agua). Ingenium, 18(35), 134-152. 
SI3EA (Sistema de Información de Eficiencia Energética y Energías Alternativas). (2010). Atlas del potencial energético de la biomasa residual en Colombia. Recuperado de https://bit.ly/3ibVLxB

Solarte, J. C.; Mariscal, J. P.; Aristizábal, B. (2017). Evaluación de la digestión y co-digestión anaerobia de residuos de comida y de poda en bioreactores a escala laboratorio. Revista ION, 30(1), 105-116. DOI: https://doi. org/10.18273/revion.v30n1-2017008 